\title{
Effect of mineral admixtures on the rheological and mechanical properties of mortars
}

\author{
Zineb DOUAISSIA ${ }^{1}$, Mouloud MERZOUD ${ }^{2}$

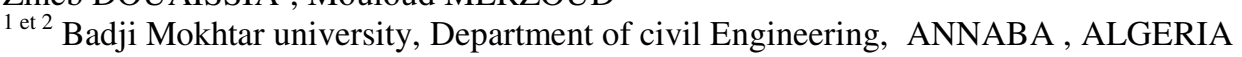

\begin{abstract}
We propose in this study to determine the role of mineral admixtures on the properties of cementitious materials in order to define a formulation method allowing an optimization of their use and the improvement of the rheological and mechanical properties of the mortars. In order to meet the objectives of this study, and to evaluate the effects that mineral admixtures may have on the properties of mortars in the fresh and hardened state, we have proposed to use a specific method based on progressive volume substitution of cement by the mineral admixtures, in order to preserve the absolute volume of all the solid constituents and the consistency for all the mixtures. The use of the mineral admixtures in the mortars also has for objective to valorize a category of natural materials or coproducts available in Algeria. As a result, the mineral admixtures chosen for this study are Blast furnace Slag, natural pozzolan and silica fume. Then the effect of the mineral admixtures on the rheological and mechanical properties of the mortars was evaluated according to the substitution rate of the cement. We applied to the studied mortars the notion of equivalent binder in order to calculate the coefficient of activity (K) defined by the two theoretical formulas Féret and Bolomey. The specific chemical action of each mixture was evaluated by analysing the variation of the coefficient of activity $\mathrm{K}$ as a function of the rate of substitution of the cement.
\end{abstract}

\section{Introduction}

Les études récentes qui ont considéré l'influence des aditions minérales sur les propriétés des matériaux de ciment [1-4] ont montré que ces additions par leur finesse et une réactivité plus ou moins significative avec le ciment, peuvent engendrer dans certains cas des modifications significatives dans les propriétés rhéologiques et mécaniques.

La norme européenne EN 206-1 [5] définit les conditions de prise en compte des additions minérales en substitution partielle du ciment dans la formulation des bétons en considérant le concept de liant équivalent et définit un coefficient forfaitaire $\mathrm{k}$ de prise en compte des additions minérales qui sont déterminées pour les cendres volantes et les fumées de silice utilisées avec un ciment du type CEM I. Dans la majorité des études publiées et conformément aux textes normatifs, les additions minérales ont été introduites dans les mélanges en substitution massique du ciment. Dans ce cas, le volume absolu du mortier augmente et le rapport volumique (eau/solide) diminue. Mais dans le cas d'une substitution volumique du ciment, le volume absolu du mortier et le rapport volumique (eau/solide) demeure sans changement. Dans ce cas, la compacité de la structure granulaire et la porosité de la matrice cimentaire des mélanges avec additions demeurent comparables à celles du mélange sans additions et seuls les effets des particules d'additions sont pris en compte dans la phase cimentaire.

Pour évaluer l'effet des additions minérales sur les caractéristiques rhéologiques et mécaniques des mortiers, nous proposons d'utiliser cette méthode basée sur la substitution volumique progressive (de 10\% jusqu'à 50\%) du ciment par du laitier granulé de haut fourneau (La), la pouzzolane Naturelle de Beni-Saf $(\mathrm{Pz})$ et la fumé de silice (Fs).

Les caractéristiques physico-mécaniques des ciments et mortiers avec la pouzzolane sont étudiées par plusieurs auteurs [6,7]. Demirboga [8] a montré en étudiant l'influence des additions minérales sur la résistance en compression des mortiers, que la densité des mortiers avec additions diminuait avec l'augmentation des additions. Autre part, il avait noté que le laitier granulé de haut fourneau engendrait une diminution de la résistance en compression à 28 jours et une amélioration à 120 jours. Ezziane et al [6] ont montré en étudiant l'efficacité du laitier d'El Hadjer par le concept du liant équivalent, que l'utilisation d'un taux élevé du laitier d'El Hadjar $(40 \%)$ lui confère un déclenchement d'activité très précoce $(2 \mathrm{j})$, seulement cette activité ne sera jamais 
achevée à long terme $(90 \mathrm{j})$. dans ce cas, un taux de $15 \%$ paraît optimal. Aichouba et al [9] ont montré en étudiant l'effet de la pouzzolane naturelle de Béni-Saf, que la substitution du ciment par la pouzzolane de $10 \%$ à $30 \%$ engendre une augmentation de la consistance normale, une diminution du temps de prise, une diminution des résistances mécaniques à jeunes âgés et une conservation de ces résistances à long terme. Colak [10] a montré que la substitution du ciment par la pouzzolane engendre une augmentation du besoin en eau des pâtes de ciment à maniabilité constante, une augmentation de la porosité de la pâte avec le taux de substitution du ciment par la pouzzolane et que les résistances en compression des mélanges à $20 \%$ de pouzzolanes sont très proches de celles sans additions.

\section{Méthodologie}

Afin d'évaluer toutes les contributions des additions aux propriétés rhéologiques et mécaniques des mortiers, nous avons été particulièrement intéressés par les propriétés suivantes:

- consistance, temps de prise, masse volumique et maniabilité.

- courbe contrainte-déformation en traction, résistances mécaniques et module d'élasticité.

- les effets des additions minérales sur le coefficient d'activité $\mathrm{K}$.

Pour atteindre cet objectif, nous avons préparé un mortier selon la norme NF EN 196-1 [11], avec une quantité d'eau ajustée pour obtenir une consistance de référence. La consistance de référence a été évaluée en mesurant l'étalement du mortier à l'état frais sur une table à secousses. Pour chaque mortier ayant acquis la consistance de référence, on a préparé des éprouvettes $4 \times 4 \times 16$.

Pour cette étude, nous avons indiqué par :

w : le volume d'eau utilisé pour obtenir un mortier avec l'efficacité de référence.

b: le volume absolu du liant dans le mortier $(b=c+a)$.

c : le volume absolu de ciment dans le mortier.

a : le volume absolu du mélange minéral dans le mortier.

\section{1 Évaluation des caractéristiques rhéologiques}

Le besoin en eau des mortiers avec additions est exprimé par la variation du rapport volumique quantité d'eau par la quantité de liant $(E / L)$. Pour apprécier les temps de début et de fin de prise des pâtes pures des liants préparés, on a effectué les essais avec l'appareil de Vicat conformément à la norme EN196-3 [12]. L'étude de la maniabilité des mortiers est assurée on étudiant deux facteurs qui sont le besoin en eau et l'étalement du mortier avec additions à l'état frais sur la table à secousses selon la norme BS EN 1015-3.

\section{2 Évaluation des caractéristiques mécaniques}

À 28 jours des essais de traction par flexion et de compression ont été effectué par la machine Zwick/Roe II Z020, dont le pilotage et l'acquisition des résultats se font par ordinateur à partir du logiciel testXpert II, à la fin de chaque essai la collecte de données nous a permis de tracer les courbes contrainte-déformation, de déterminer les résistances à la traction et à la compression ainsi de déduire les modules d'élasticité.

\subsection{Evaluation du coefficient d'activité $\mathrm{K}$ :}

L'évaluation du coefficient d'activité $\mathrm{K}$ pour une addition minérale incorporée dans un mélange cimentaire, peut se faire en appliquant aux mortiers étudiés les principes des formules théoriques de Féret et Bolomey.

\subsubsection{Application de la formule de Féret:}

La formule de Féret exprime la résistance en compression par la relation suivante :

$$
R_{e}=G_{F} \times f_{m e}\left(\frac{v}{c+i+v}\right)^{2}
$$

$c, e$ et $v$ désignent respectivement, les volumes du ciment, d'eau et d'air occlus dans le mortier sans additions en $\left(1 / \mathrm{m}^{3}\right)$.

$G_{F}$ désigne un coefficient adimensionnel qui dépend de la nature des agrégats.

$f_{m c}$ désigne la résistance en compression du mortier normal au même âge en (MPa).

nous pouvons nous baser sur la notion de liant équivalent définie par la norme européenne EN 206-1, comme suit :

$$
L_{\text {squ }}=C+k F
$$

$C$, désigne la quantité de ciment dans le béton $\left(\mathrm{kg} / \mathrm{m}^{3}\right)$, $F$, désigne la quantité d'addition dans le béton $\left(\mathrm{kg} / \mathrm{m}^{3}\right)$, $k$, désigne un coefficient de prise en compte des additions minérales dans le liant.

Le facteur d'activité $K_{F}$ peut être déduit à partir du modèle prédictif de Féret, comme suit :

$$
K_{F}=\frac{\varepsilon_{r}(\varepsilon+w) \sqrt{R_{\varepsilon} / R_{g r}}}{f\left[c _ { r } \left(1-\sqrt{\left.\left.R_{g} / R_{g}\right)+\left(\varepsilon_{p}+v\right)\right]}\right.\right.}-\frac{\varepsilon}{f}
$$

\subsubsection{Application de la formule de Bolomey :}

En se basant sue le modèle prédictif de Bolomey, la résistance en compression est exprimée par la relation suivante :

$$
R_{e}=G_{B} \cdot f_{e}\left(\frac{c}{E+V}-0.5\right)
$$

$c$, $e$ et $v$, désignent respectivement, la masse du ciment, la masse d'eau et la masse d'eau équivalente au volume d'air occlus dans le mélange en $\left(\mathrm{kg} / \mathrm{m}^{3}\right)$.

$G_{B}$ désigne un coefficient adimensionnel qui dépend de la nature des agrégats.

$f_{c}$ désigne la résistance en compression du mortier normal au même âge en (MPa).

En appliquant aux mortiers étudiés, la notion du liant équivalent, le facteur d'activité $K_{B}$ peut être déduit à partir du modèle prédictif de Bolomey, comme suit :

$K_{E}=\frac{R_{\varepsilon} \cdot C_{r}(E+V)}{R_{c p} \cdot F\left(E_{p}+V_{p}\right)}-\frac{C}{F}+\frac{E+V}{2 F}\left(1-\frac{R_{c}}{R_{c r}}\right) \ldots \ldots \ldots$ Avec :

$$
\begin{aligned}
& C_{r}=\rho_{C^{n}} C_{r} \\
& C=\rho_{C^{n}} C \\
& F=\rho_{F} \cdot f
\end{aligned}
$$


$\rho_{C}$ et $\rho_{F}$ désignent respectivement, les masses volumiques absolues du ciment et de l'addition.

$c, c_{\mathrm{r}}$ et $f$ désignent respectivement, les volumes de ciment et d'additions dans le mortier.

\section{Matériaux}

\subsection{Ciment}

Le ciment utilisé est un ciment Portland du type CPA CEMI 52.5R conformément a la norme NF EN 197-1 [13]. Sa masse volumique et sa surface spécifique sont respectivement $3,15 \mathrm{~g} / \mathrm{cm}^{3}$ et $440 \mathrm{~m}^{2} / \mathrm{kg}$.

\subsection{Sable}

Le sable utilisé est un sable normalisé, le sable est conditionné en sachets de polyéthylène contenant chacun $1350 \pm 5$ g conformément aux exigences de la norme EN 196-1.

\subsection{Additions minérales}

Pour cette étude, il a été donc utilisé trois additions minérales différentes par leurs natures minéralogiques, morphologiques et chimiques : une addition de pouzzolane $(\mathrm{Pz})$, une addition de laitier granulé vitrifié moulu de haut fourneau (La) et une addition de fumée de silice (Fs), (tableau.1).

Tableau.1: Caractéristiques physiques des additions minérales

\begin{tabular}{|l|c|c|c|}
\hline \multicolumn{2}{|c|}{ Additions } & $\begin{array}{c}\text { Masse volumique } \\
\text { Absolue }\left(\mathrm{Kg} / \mathrm{m}^{3}\right)\end{array}$ & $\begin{array}{c}\text { Surface spécifique } \\
\text { Blaine }\left(\mathrm{cm}^{2} / \mathrm{g}\right)\end{array}$ \\
\hline laitier & $\mathrm{La}$ & 2800 & $2900 \pm 120$ \\
\hline pouzzolane & $\mathrm{Pz}$ & 2650 & $3500 \pm 200$ \\
\hline fumée de silice & $\mathrm{Fs}$ & 2240 & $15.10-9$ \\
\hline
\end{tabular}

\section{Résultats}

\subsection{Effets des Additions minérales sur les caractéristiques rhéologiques des mortiers}

\subsubsection{Consistance et temps de prise :}

On peut constater sur la figure. 1 que l'ajout des additions minérales implique une augmentation de $3 \%$ de la quantité d'eau pour obtenir une consistance normale.

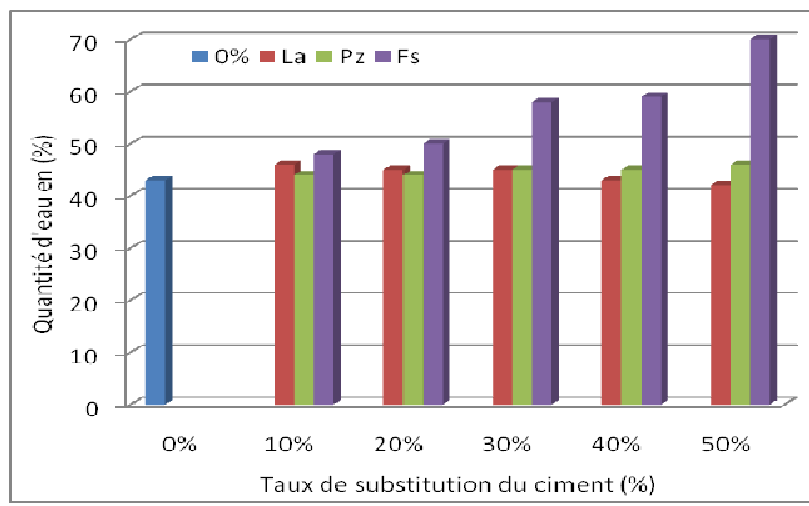

Figure 1 : Variation de la consistance normale des pâtes, en fonction du taux de substitution du ciment par les additions minérales.

Les figures 2 et 3 traduisent les temps de début et fin de prise des pâtes, en fonction du taux de substitution du ciment par les additions minérales. On constate une augmentation des temps de début de prise par rapport à celles de la pâte témoin $(0 \%)$ a eu pour effet de retarder l'hydratation du ciment (phase de ralentissement), ce retard de la prise est attribué principalement à la baisse de la teneur de ciment en $\left(\mathrm{C}_{3} \mathrm{~A}\right)$ qui est responsable $\mathrm{du}$ raidissement précoce.

\subsubsection{Masse volumique des mortiers frais :}

Les courbes de la variation de la masse volumique des mortiers à l'état frais présentées par la figure 4, sont décroissantes avec le taux de substitution, un optimum est observé pour des taux de $10 \%$ et $20 \%$.

En général, les masses volumiques apparentes des mortiers avec additions minérales à l'état frais sont directement liées au besoin en eau et suit une variation opposée.

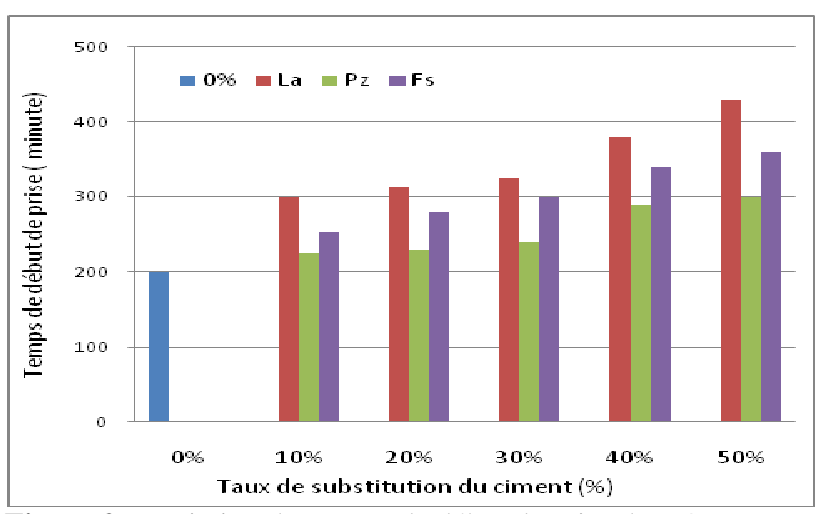

Figure 2: Variation des temps de début de prise des pâtes, en fonction du taux de substitution du ciment par les additions minérales.

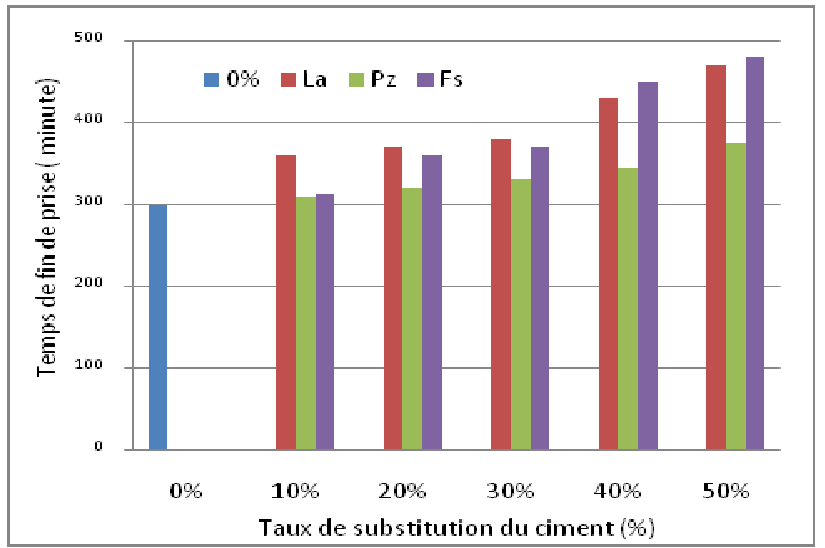

Figure 3 : Variation des temps de fin de prise des pâtes, en fonction du taux de substitution du ciment par les additions minérales. 


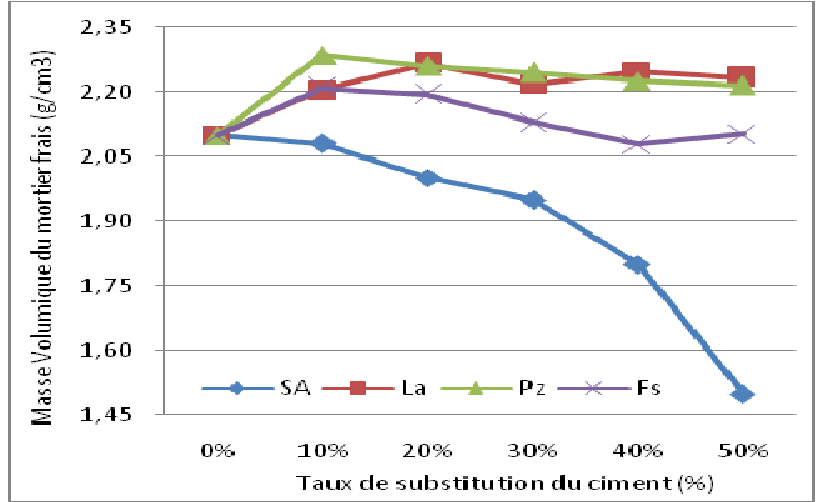

Figure 4 : Variation de la masse volumique des mortiers à l'état frais, à même consistance en fonction du taux de substitution du ciment par les additions minérales.

\subsubsection{Maniabilité :}

Nous constatons sur la figure 5, que l'étalement du mortier frais avec la pouzzolane $(\mathrm{Pz})$, crô̂t jusqu'à $10 \%$ du taux de substitution, puis décroît avec l'augmentation de la quantité d'addition dans le mélange.

Les mortiers frais avec du laitier (La) et de la fumée de silice (Fs), présentent un étalement qui décroît directement avec l'augmentation de la quantité d'addition dans le mélange. Le mortier sans additions (SA) présente un comportement différent. En effet la substitution du ciment par le sable engendre une amélioration continue de l'ouvrabilité.

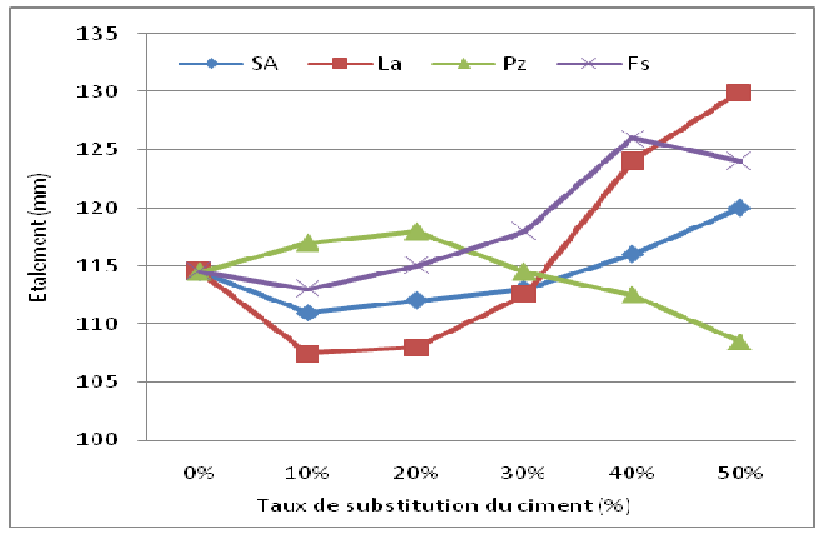

Figure 5 : Variation de l'étalement sur la table à secousses des mortiers, en fonction du taux de substitution du ciment par les additions minérales.

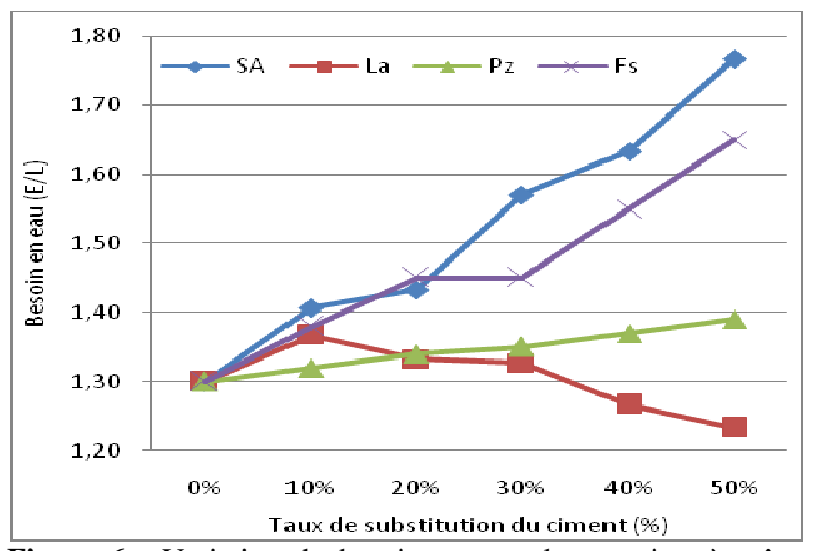

Figure 6 : Variation du besoin en eau des mortiers à même consistance, en fonction du taux de substitution du ciment par les additions minérales
Nous constatons sur la figure 6 que les mortiers avec la pouzzolane présentent un besoin en eau plus faible que celui du mortier de référence pour des taux inférieurs à $20 \%$. À plus forts taux de substitution, le besoin en eau croît avec l'augmentation de la quantité d'addition dans le mélange, mais reste proche de celui du mortier de référence.

Les mortiers avec du laitier présentent un besoin en eau qui croit avec le taux de substitution du ciment. Cette différence de comportement est probablement due à l'irrégularité et à l'aspect poreux de ses particules, qui n'arrivent plus à remplir les pores du squelette granulaire ou absorbent des quantités d'eaux complémentaires qui peut se traduire par un plus fort besoin en eau, à même consistance.

On peut également noter que les mortiers avec fumée de silice présentent les besoins en eau les plus importants en accord avec sa grande finesse, ce besoin en eau est d'autant plus important que le taux de substitution du ciment est grand. Les mortiers sans additions présentent un besoin en eau décroissant.

\subsection{Effets des Additions minérales sur les caractéristiques mécaniques des mortiers:}

\subsubsection{Courbes contraintes - déformations en traction par flexion :}

La comparaison des courbes contrainte-déformation en traction par flexion des mortiers est présentée par les figures 7,8 et 9 .

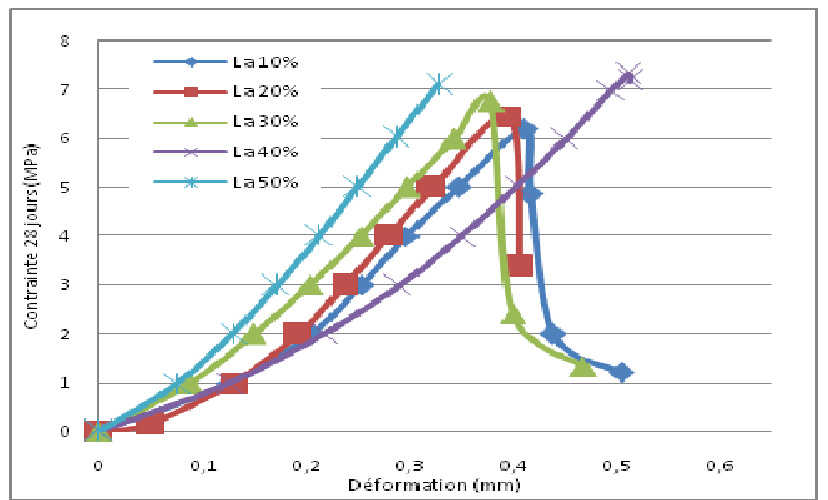

Figure 7 : courbes contrainte-déformation en traction par flexion des mortiers en fonction du taux de substitution du ciment par le laitier. 


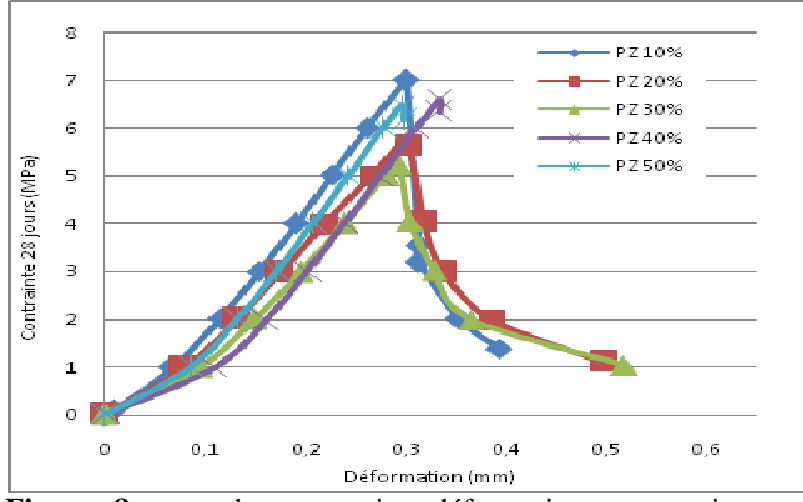

Figure 8 : courbes contrainte-déformation en traction par flexion des mortiers en fonction du taux de substitution du ciment par la pouzzolane

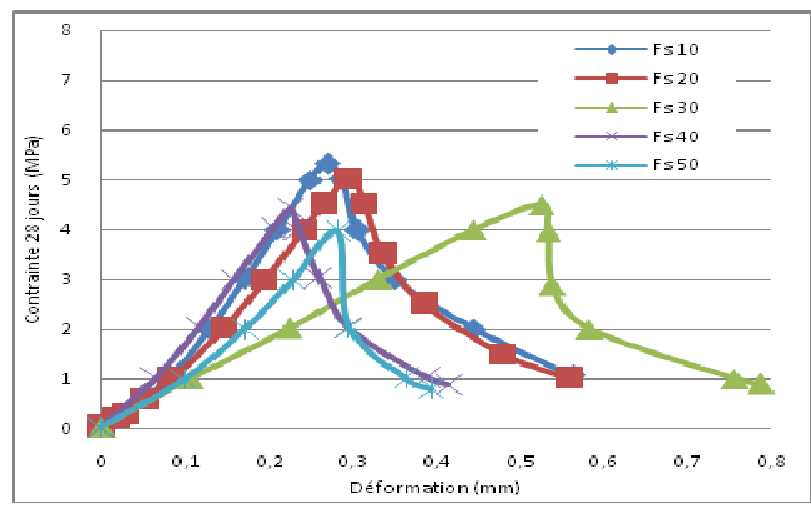

Figure 9 : cou rbes contrainte-déformation en traction par flexion des mortiers en fonction du taux de substitution du ciment par la fumée de silice .

Nous observons que toutes les courbes présentent un comportement linéaire élastique jusqu'à environ $30 \%$ de la résistance maximale. Au-delà de la valeur limite d'élasticité, les courbes enregistrent une augmentation graduelle en courbure évaluée autour de 75 à $90 \%$ de la résistance ultime. Au-delà du pic, la courbe présente une partie descendante (déformation contrôlée) jusqu'à la rupture. L'addition de fumée de silice améliore considérablement la rigidité des mortiers, tandis que le laitier et la pouzzolane peuvent engendrer une certaine fragilité quoiqu'ils donnent aussi les valeurs de résistances à la traction par flexion les plus ou moins importantes que la fumée de silice.

\subsubsection{Résistances mécaniques :}

La variation des résistances à la traction et en compression à 28 jours est représentée par les figures 10 et 11 .

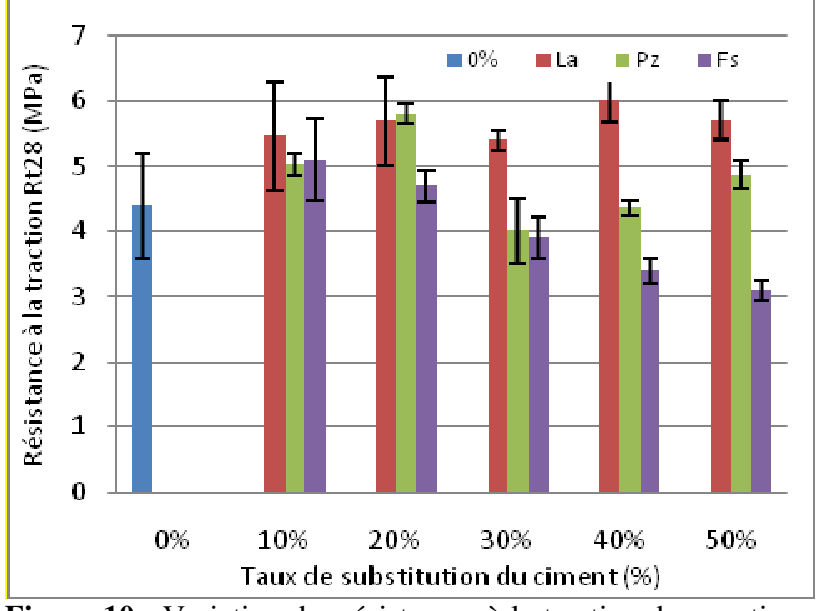

Figure 10: Variation des résistances à la traction des mortiers, en fonction du taux de substitution du ciment par les additions minérales.

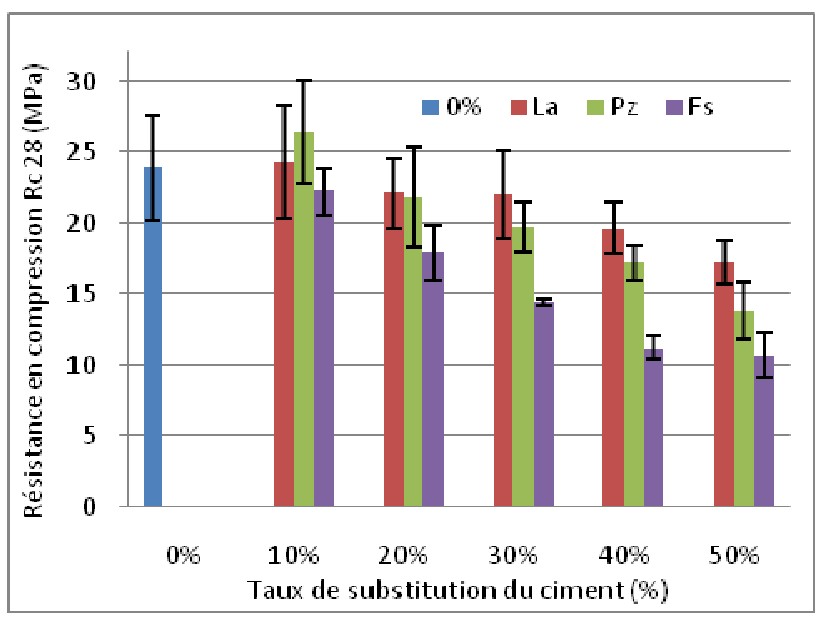

Figure 11 : Variation des résistances en compression des mortiers, en fonction du taux de substitution du ciment par les additions minérales.

Nous remarquons que les résistances à la traction croissent pour les faibles taux de substitution du ciment , $20 \%$ pour le laitier et la pouzzolane et $10 \%$ pour la fumée de silice, puis commencent à décroître.

Les résistances en compression dépendent de taux de substitution du ciment. Des résistances importantes sont obtenues pour les mortiers avec le taux (10\%), avec des valeurs dépassant celui de la référence sans additions minérales. Néanmoins, la tendance générale est au delà de $20 \%$ de substitution conduit à une baisse de résistance en compression, principalement en raison de l'effet de dilution, qui correspond bien avec les résultats obtenus par d'autres auteurs [8].

\subsubsection{Module d'élasticité E :}

Les variations du module l'élasticité à 28 jours sont représentées par la figure 12. Nous remarquons que les modules d'élasticité en compression $\mathrm{E}_{28}$ croissent pour les faibles taux de $20 \%$ à $30 \%$ pour le laitier et $10 \%$ pour la pouzzolane et de $10 \%$ à $20 \%$ pour la fumée de silice, puis commencent à décroître proportionnellement aux taux de substitution. 


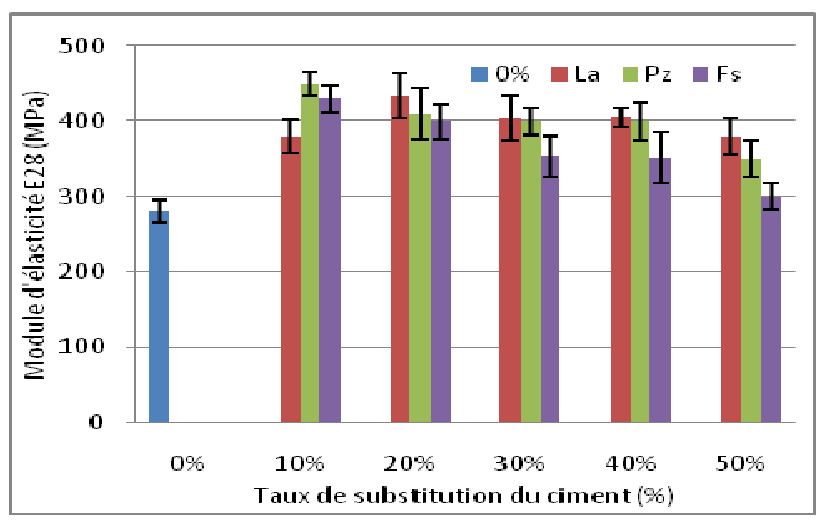

Figure 12: Variation du Module l'élasticité des mortiers, en fonction du taux de substitution du ciment par les additions minérales.

\subsection{Effets des Additions minérales sur le coefficient d'activité $\mathrm{K}$ :}

\subsubsection{Application de la formule de Féret :}

Les variations des facteurs d'activité chimique des additions calculé par la formule de Féret, est représentée par la figure 13, à faibles taux de substitution, la fumée de silice présente à $10 \%$ facteur d'activité le plus important parmi toutes les additions utilisées. Il atteint une valeur de 1,99. Les facteurs d'activité de la pouzzolane sont les plus faibles en raison de l'atténuation des effets physicochimiques qui sont d'autant plus faibles que la finesse de l'addition est petite. Le laitier présente des facteurs d'activité intermédiaires, avec une meilleure activité à $20 \%$ pour laquelle il atteint une valeur de 1,11 .

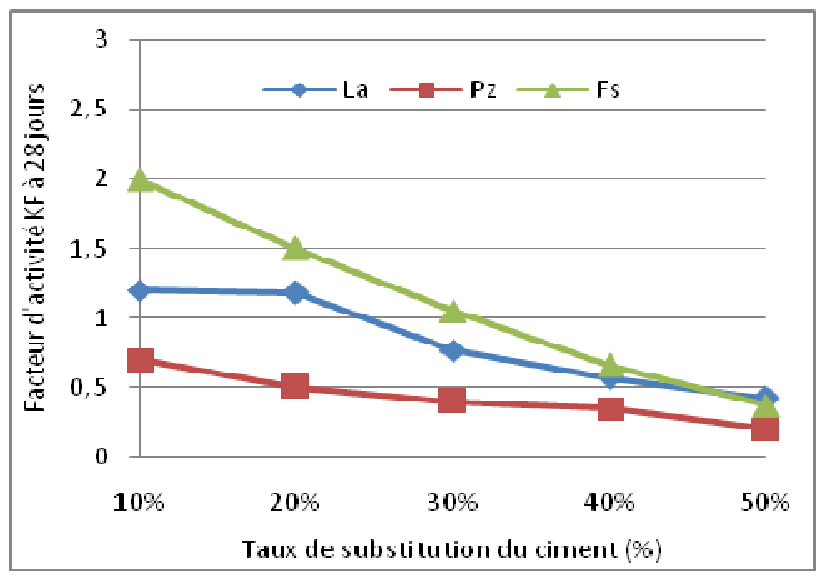

Figure 13 : Variation du coefficient d'activité K, calculé à partir de la formule de Féret, en fonction du taux de substitution du ciment par les additions minérales.

\subsubsection{Application de la formule de Bolomey :}

Les variations des facteurs d'activité chimique des additions à 28 jours pour la formule de Bolomey, sont représentées par les figures 14 .

Dans le cas de la formule de Bolomey, à faibles taux de substitution du ciment, la fumée de silice présente à $10 \%$ du taux de substitution le facteur d'activité le plus important parmi toutes les additions utilisées. Il atteint une valeur de 2,75. Les facteurs d'activité de la pouzzolane sont les plus faibles en raison de l'atténuation des effets physicochimiques. Le laitier présente des facteurs d'activité intermédiaires, avec une meilleure activité à $20 \%$ du taux de substitution du ciment, pour laquelle ils atteint la valeur de 1,30.

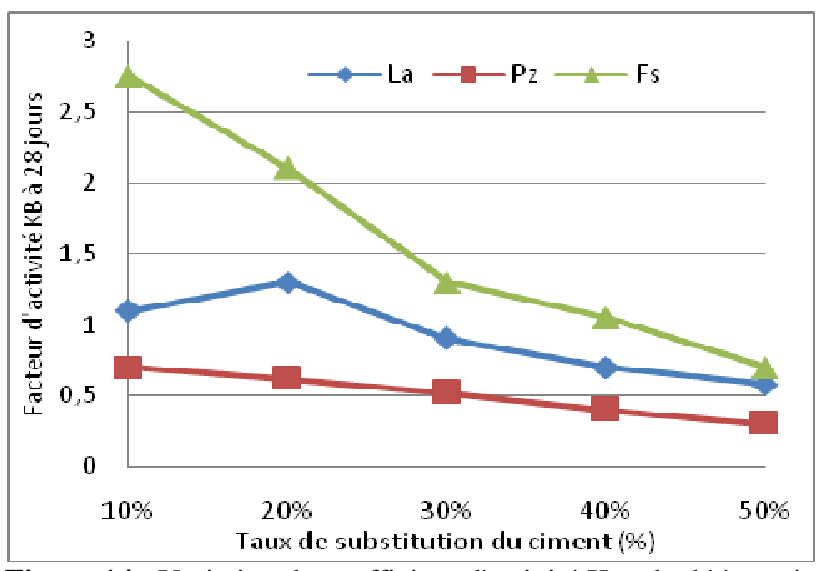

Figure 14 : Variation du coefficient d'activité $K$, calculé à partir de la formule de Bolomy, en fonction du taux de substitution du ciment par les additions minérales.

Néanmoins, il est important de noter que les facteurs d'activité évalués à partir de la formule de Bolomey sont beaucoup trop surestimés pour le cas de la fumée de silice par rapport à ceux calculés à partir de la formule de Féret.

\section{Conclusions}

L'approche appliquée consiste en une substitution progressive du ciment par des additions minérales dans des mortiers dont la maniabilité et le volume absolu de toutes les phases constitutives solides sont restés constants.

Les résultats obtenus ont montré que:

- le besoin en eau des mortiers varie sensiblement avec la nature minéralogique et les caractéristiques granulaires du mélange minéral incorporé, et pour les faibles taux de substitution du ciment, elle est directement liée aux phénomènes d'empilage granulaire, donc à la compacité des mélanges frais.

- l'effet des additions minérales se limite principalement à une accélération du processus d'hydratation de ciment à un jeune âge. L'action contraire de la fumée de silice est probablement due aux réactions pouzzolaniques relativement tardives avec le ciment.

Ensuite, les résistances se développent avec des taux de substitution faibles (à proximité de 10\% ). Ensuite, elles commencent à diminuer, à l'exception du mortier avec de la fumée de silice qui diminue directement sans aucune amélioration.

- L'activité chimique des additions à 28 jours pour les mortiers avec additions, a été évaluée en traçant la variation du facteur d'activité en fonction du taux de substitution du ciment par l'addition, en utilisant la notion du liant équivalent définie par la norme EN 206-1 et en déduisant le facteur d'activité chimique à partir des formules théoriques de Féret et de Bolomey.

Les facteurs d'activités chimiques à 28 jours, des différentes additions sont très variables. Ils dépendent de l'âge du mortier avec additions, de la formule de résistance adoptée, de la nature minéralogique et 
chimique de la quantité et des caractéristiques granulaires de l'addition incorporée. Ils sont encore plus forts dans le cas de la formule théorique de Bolomey que dans le cas de la formule de Féret.

\section{References}

1. Logbi Abdelaziz. "Effet de l'incorporation des ajouts minéraux sur les propriétés physico-mécaniques du béton", Thèse de Magister, l'ENP-Alger (Juin 1999).

2. Chabi. S, Mezghiche. B, et Guettala.H; "Etude de l'influence des additions minérales actives sur le comportement mécaniques des ciments et mortiers" Courrier du Savoir - Nº5, pp. 03-08, (Juin 2004).

3. A. Kerbouche, M. Mouli, L. Laoufi, Y. Senhadji et S. Benosmane; "Influence des ajouts minéraux sur les résistances mécaniques des mortiers". SBEIDCO -1st International Conference on sustainable Built Environment Infrastructures in Developing Countries-ENSET, Oran-Algeria, (2009).

4. Amouri Chahinez, Houari Hacène; "les performances des mortiers avec differentes additions minerales" SBEIDCO -1st International Conference on Sustainable Built Environment Infrastructure in Developing in Developing Countries ENSET-OranAlgeria, (2009)

5. AFNOR NF EN 206-1 Béton-Partie 1: spécification performances, production et conformité- (Avril 2004)

6. Ezziane K., Bougara A., Kassoul A., ""Efficacité du laitier d'El Hadjar par le concept du liant équivalent", 2ème colloque Maghrébin en Génie Civil, p. 87-96, Biskara, Algérie, 10-11 (décembre 2002).

7. 7.Boudchicha A., Utilisation des additions minérales et des adjuvants fluidifiants pour l'amélioration des propriétés rhéologiques et mécaniques des bétons, Thèse de doctorat, université de Constantine, (2007).

8. Demirboga R., Influence of mineral admixtures on thermal conductivity and compressive strength of mortar, Energy and building Vol. 35, p. 189-192, (2003).

9. Aichoube A., Ghrici M., Benaissa A., "Effect de la pouzzolane naturelle de Béni-Saf sur les propriétés d'un ciment à base de calcaire", Colloque Méditerranéen sur les Matériaux CMEDIMAT, Oran, Algérie, 6-7 (décembre 2005).

10. Colak A., "Characteristics of pastes from a Portland cement containing different amounts of natural pouzzolan", Cement and Concrete Research, Vol. 33, p. 585-593, (2003).

11. AFNOR NF EN 196-1 Méthodes d'essais des ciments- Partie 1 : Détermination des résistances mécaniques, (avril 2006).

12. NF EN 196-3 (P15-473) : Méthodes d'essais des ciments - partie 3 : Détermination du temps de prise et de la stabilité.F. De Lillo, F. Cecconi, G. Lacorata, A. Vulpiani, EPL, 84 (2008)
13. AFNOR EN 197-1 Ciment - Partie 1 : Composition, spécifications et critères de conformité, (février 2001). 